\title{
Cadherin 13: Human cis-Regulation and Selectively Altered Addiction Phenotypes and Cerebral Cortical Dopamine in Knockout Mice
}

\author{
Jana Drgonova, ${ }^{1}$ Donna Walther, ${ }^{1}$ G Luke Hartstein, ${ }^{1}$ Mohammad O Bukhari, ${ }^{2}$ Michael H Baumann, ${ }^{2}$ \\ Jonathan Katz, ${ }^{2}$ F Scott Hall, ${ }^{1}$ Elizabeth R Arnold, ${ }^{1}$ Shaun Flax, ${ }^{3}$ Anthony Riley, ${ }^{3}$ Olga Rivero, ${ }^{4}$ \\ Klaus-Peter Lesch, ${ }^{4}$ Juan Troncoso, ${ }^{5}$ Barbara Ranscht, ${ }^{6}$ and George R Uhi ${ }^{1,7}$
}

${ }^{1}$ Molecular Neurobiology; ${ }^{2}$ Medicinal Chemistry, National Institute on Drug Abuse, Intramural Research Program, Baltimore, Maryland, United States of America; ${ }^{3}$ Department of Psychology, American University, Washington, DC, United States of America; ${ }^{4}$ Translational Neurobiology, Department of Psychiatry, University of Würzburg, Würzburg, Germany; ${ }^{5}$ Division of Neuropathology, Johns Hopkins School of Medicine, Baltimore Maryland, United States of America; ${ }^{6}$ Sanford-Burnham Medical Research Institute, La Jolla, California, United States of America; and ${ }^{7}$ Research, New Mexico VA Healthcare System, Albuquerque, New Mexico, United States of America

\begin{abstract}
The cadherin 13 (CDH13) gene encodes a cell adhesion molecule likely to influence development and connections of brain circuits that modulate addiction, locomotion and cognition, including those that involve midbrain dopamine neurons. Human CDH13 mRNA expression differs by more than $80 \%$ in postmortem cerebral cortical samples from individuals with different $\mathrm{CDH} 13$ genotypes, supporting examination of mice with altered $\mathrm{CDH} 13$ expression as models for common human variation at this locus. Constitutive $\mathrm{CDH} 13$ knockout mice display evidence for changed cocaine reward: shifted dose response relationship in tests of cocaine-conditioned place preference using doses that do not alter cocaine-conditioned taste aversion. Reduced adult $\mathrm{CDH} 13$ expression in conditional knockouts also alters cocaine reward in ways that correlate with individual differences in cortical $\mathrm{CDH} 13$ mRNA levels. In control and comparison behavioral assessments, knockout mice display modestly quicker acquisition of rotarod and water maze tasks, with a trend toward faster acquisition of 5-choice serial reaction time tasks that otherwise displayed no genotype-related differences. They display significant differences in locomotion in some settings, with larger effects in males. In assessments of brain changes that might contribute to these behavioral differences, there are selective alterations of dopamine levels, dopamine/metabolite ratios, dopaminergic fiber densities and mRNA encoding the activity dependent transcription factor npas 4 in cerebral cortex of knockout mice. These novel data and previously reported human associations of $C D H 13$ variants with addiction, individual differences in responses to stimulant administration and attention deficit hyperactivity disorder (ADHD) phenotypes suggest that levels of $\mathrm{CDH} 13$ expression, through mechanisms likely to include effects on mesocortical dopamine, influence stimulant reward and may contribute modestly to cognitive and locomotor phenotypes relevant to ADHD.
\end{abstract}

Online address: http://www.molmed.org

doi: $10.2119 / \mathrm{molmed} .2015 .00170$

\section{INTRODUCTION}

Cadherin 13 (CDH13) encodes a glycosylphosphatidyl inositol-anchored cell adhesion molecule whose variation is likely to alter connections between neurons in which it is expressed $(1,2)$.
CDH13 mRNA is prominently expressed by ventral tegmental area and substantia nigra pars compacta neurons that are implicated in reward, locomotor control and cognitive modulation $(3,4)$. This localization increases interest in effects of $\mathrm{CDH} 13$

Address correspondence to George Uhl, Research, Building 66, Room 127, New Mexico VA Healthcare System, 1501 San Pedro SE, Albuquerque, New Mexico 87111 , USA. Phone: +1-505-265-1711, x4712; E-mail: George.Uhl@va.gov.

Submitted July 15, 2015; Accepted for Publication July 29, 2016; Published Online (www.molmed.org) August 18, 2016.

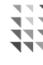

Feinstein Institute for Medical Research Northwell Health

variation on dopaminergic brain systems and dopamine-associated behaviors.

Experiments that test the consequences of modifying $\mathrm{CDH} 13$ expression are also motivated by human molecular genetic studies. For addiction vulnerability and attention deficit hyperactivity disorder (ADHD), the CDH13 locus is marked by groups of clustered, nearby single nucleatide polymorphisms (SNPs) that display associations with $10^{-2}>p>10^{-8}$ statistical significance in numerous independent case versus control genome wide association (GWAS) studies (5-15). CDH13 associations with the numbers of cigarettes smoked per day, reward reported after 
oral modest amphetamine doses and effects of individuals' first five uses of alcohol are also described $(7,16,17)$.

PubMed searches for cadherin 13 identify nine publications relating to normal brain, most of which relate to abovementioned $\mathrm{CDH} 13$ associations with substance dependence or ADHD. Two reports describe different $\mathrm{CDH} 13$ knockout mice, one with altered hippocampal function and modest influences on freezing and reversal learning during fear conditioning and Barnes maze tests $(19,20)$. We now report the correlation of human $\mathrm{CDH} 13$ genotypes with individual differences in levels of $\mathrm{CDH} 13$ mRNAs in postmortem cerebral cortical samples. This finding, and failure to identify common disease-associated missense $C D H 13$ variants, indicate that altered levels of $\mathrm{CDH} 13$ expression may represent a major genetic contribution to human interindividual differences at this locus. These human results help to motivate a variety of studies in mice with altered CDH13 expression.

In this paper, we study mice with constitutively altered and with adult (via conditional knockout) reductions in levels of CDH13 mRNA. We test these mice using conditioned place preference (CPP), a model of rewarding influences from drugs that has been highly validated in pharmacologic studies (18). Results support influences of $\mathrm{CDH} 13$ variation on addiction-related phenotypes and the possibility that drugs that modify activities of CDH13 might be useful when given to adults. Conclusions from CPP tests are buttressed by results of evaluations of other phenotypes, including tests of motor abilities (reductions might confound CPP results), learning/ memory (reductions might confound CPP results), aversive features caused by cocaine (which might provide an alternative explanation for CPP results to "altered reward"), impulsivity (which might fit with ADHD influences but confound CPP results) and anxiety (which might confound CPP results). We do report more subtle influences of CDH13 knockout on ADHD-related tests of locomotion in male mice and on performance during early acquisition trials for complex tasks.

When we test levels of monoamines in brains of the knockout mice as possible contributors to observed behavioral differences, we identify selectively altered cerebral cortical dopamine/ metabolite ratios and differences in dopaminergic fiber densities. These novel data, and recent work by others, provide substantial a posteriori likelihood that common human $\mathrm{CDH} 13$ "level of expression" variation contributes to differences in brain connections that are reflected in phenotypes relevant to addiction, stimulant actions, locomotor control and learning of complex tasks.

\section{MATERIALS AND METHODS}

Human brain samples for studies of CDH13 mRNAs in individuals with different CDH13 SNP alleles were obtained under protocols overseen by the Johns Hopkins (19\% of samples) and University of Maryland ( $81 \%$ of samples) Institutional Review Boards. Mouse breeding and all experiments were performed under protocols approved by the NIDA-IRP Institutional Animal Care and Use Committee that complied with the Guide for the Care and Use of Laboratory Animals (8th edition, NRC 2011).

Human CDH13 mRNA correlation with CDH13 genomic markers was sought in frontal cortical autopsy samples (Johns Hopkins and University of Maryland brain banks) from European American individuals who lacked gross neuropathology. We focused on this genetic background and this brain region since this focus allow us to assemble a large a group of brain samples not possible using material from other brain regions or individuals with different racial/ ethnic backgrounds. Most common causes of death were accidents/multiple trauma, cardiovascular disease and pulmonary embolisms. Average time to freezing was14 h. Brains came from individuals who were $50.5 \%$ female and with an average age 42 . RNAs were prepared with the RNeasy lipid tissue mini kits (Qiagen), cDNA synthesized with SuperScript III first strand synthesis supermix (Invitrogen) and levels of mRNAs assessed by quantitative real-time polymerase chain reaction (RT-PCR) using SybrGreen master mix (Applied Biosystems) according to the manufacturer's protocol using oligonucleotide primers (sequences available from authors on request) that targeted the dominant long CDH13 mRNA isoform 1 (www.ncbi.nlm.nih.gov/IEB/ Research/Acembly) and the reference genes glyceraldehyde-3-phosphate dehydrogenase (GAPDH), hypoxanthine phosphoribosyltransferase 1 (HPRT1) and ubiquitin C (UBC).

CDH13 SNP genotyping was performed in DNA extracted from brain samples using Qiagen kits genotyped in multiplexed Sequenom panels (see Supplementary Materials).

Constitutive CDH13 knockout mice were produced by standard methods using embryonic stem cells from 129/sv mice and backcrossed for more than 15 generations to C57BL/6J mice (19). Mice from heterozygote $\times$ heterozygote matings were genotyped as described (19) and tested at $90 \pm 43 \mathrm{~d}$ of age.

Conditional CDH13 knockout mice. $\mathrm{CDH}_{13}{ }_{\text {loxp }}$ mice were produced as described (20) and bred with UBC-Cre/ ERT2 mice (Jackson Laboratory; \#008085) to obtain homozygous CDH13 $3_{\text {loxP } / \text { loxp }}$; UBC-Cre/ERT2 \pm and CDH13 $3_{\text {loxP/loxp; }}$; UBC-Cre/ERT2_/- mice.

CDH13 deletion was induced by treating adult mice (average 89 -d-old) with tamoxifen (Sigma; $200 \mathrm{mg} / \mathrm{kg} /$ day intraperitoneal (i.p.)) for five days approximately two weeks prior to testing.

Cocaine-conditioned place preference was assessed as described $(21,22)$. Briefly, preferences for one side of the apparatus were assessed, four 20 min conditioning trials conducted (two cocaine, two saline) and preferences were assessed again $24 \mathrm{~h}$ after the last conditioning session. Differences between times spent on the drug-paired side during the post- versus pre-tests were noted.

Cocaine-conditioned taste aversion consisted of habituation, conditioning 
and two-bottle testing, as described (23). After acclimation, handling and $20 \mathrm{~min} / \mathrm{d}$ access to water, mice were given four series of 20-min conditioning sessions during which they were provided access to $1 \mathrm{~g} / \mathrm{L}$ saccharin solution, then injected within 20 min with $0,5,10$ or $20 \mathrm{mg} / \mathrm{kg}$ cocaine i.p., then $2 \mathrm{~d}$ of water recovery. Saccharin solution consumed when mice were given access to both saccharin and water for $20 \mathrm{~min}$ in a two-bottle-conditioned taste avoidance test was assessed.

Locomotion was recorded for $60 \mathrm{~min}$ in $42 \times 42 \mathrm{~cm}$ dark, soundattenuated boxes to which the mice had not been previously exposed and during conditioned place preference sessions of 20 min pretest (access to both halves of the $20 \times 40$ conditioning apparatus), conditioning (access to only $20 \times 20 \mathrm{~cm}$ half of the apparatus) and test (access to $20 \times 40 \mathrm{~cm}$ ) sessions. Distances traveled were calculated from infrared beam breaks (Optovarimax ATS System) (22).

Motor coordination and learning were tested once per day over three consecutive days on a rotarod that accelerated from 4 to $40 \mathrm{rpm}$ over $5 \mathrm{~min}$.

Memory and learning were evaluated in a Morris water maze (24). A black 90-cm diameter pool filled with opaque $22^{\circ} \mathrm{C}$ water contained a $9-\mathrm{cm}$ platform in the center of one quadrant that was visible for the first six trials and hidden $0.5 \mathrm{~cm}$ below the water level for subsequent trials. Mice received two trials, each lasting a maximum of $60 \mathrm{~s}$, separated by a $15 \mathrm{~s}$ rest period on the platform. They were returned to home cages for $4 \mathrm{~h}$, then given an additional 2-trial session. After acquisition, defined by an average latency to reach the platform of less than $10 \mathrm{~s}$, the platform was removed and a $60 \mathrm{~s}$ probe trial conducted. Probe trial data including the path of the subject in the pool and the time spent in each quadrant were analyzed with Ethovision software (Noldus). After probe trials, the platform was placed in the opposite quadrant to assess reversal learning using the same procedure.

Five choice serial reaction time/learning was tested in light-proof, ventilated sound attenuated operant chambers (MED-NP5L; Med-Associates) with masking white noise, five wall holes that could be illuminated and an opposite-wall food tray that received 45-mg food pellets. Mice initially received a pellet if they poked the appropriate hole during the 60-s period during which it was illuminated (FR1 schedule). Any response during the response period ended the trial, which was followed by a 5-s intertrial interval. The 60-s stimulus duration used for the first block of trials was decreased to $30,15,7.5$ and $4 \mathrm{~s}$ in successive weekly blocks of trials. Sessions ended after 50 food presentations or $40 \mathrm{~min}$.

Anxiety was evaluated by testing time for emergence from a dark box $(18 \times 18 \mathrm{~cm})$ through a $5-\mathrm{cm}$ opening into a brightly illuminated $18 \times 18 \mathrm{~cm}$ field, and thigmotaxis, time spent in near walls versus near the center of the $42 \times 42 \mathrm{~cm}$ open field apparatus under red light conditions.

Monoamine/metabolite levels were examined in extracts of ventral midbrain, hippocampus, striatum and cerebral cortical specimens that were dissected on ice, frozen and stored at $-80^{\circ} \mathrm{C}$. Tissue samples were weighed, homogenized in $4^{\circ} \mathrm{C} 0.1 \mathrm{~N} \mathrm{HClO}_{4}$ and concentrations of dopamine (DA), serotonin (5-HT) and their respective metabolites: 3,4dihydroxyphenylacetic acid (DOPAC), homovanillic acid (HVA) and 5-hydroxyindoleacetic acid (5-HIAA) quantified using high-pressure liquid chromatography with a $\mathrm{C}_{18}$ column, mobile phase $50 \mathrm{mmol} / \mathrm{L}$ sodium phosphate monobasic, $2501 \mathrm{M} \mathrm{Na}_{2}$ ethylenediamine tetraacetic acid (EDTA), $0.03 \%$ sodium octanesulfonic acid $/ 25 \%$ methanol (final $\mathrm{pH} 2.75$ ), Coulochem III detector (Thermo Scientific Dionex) and Empower 2 software Waters Corp.) as described (25). Peak heights of unknowns were compared to those of standards; lower limits of detection were $1 \mathrm{pg} / 20 \mu \mathrm{L}$.

Cortical dopamine fiber densities were examined in $50-\mu \mathrm{m}$ coronal vibratome sections cut through six levels of frontal cortices (bregma +1.38 to $+0.68 \mathrm{~mm}$ ) of pentobarbital-anesthetized mice perfused through their left ventricles with $4 \%$ depolymerized paraformaldehyde in phosphate-buffered saline (PBS). Brains were postfixed for $4 \mathrm{~h}$ at $4^{\circ} \mathrm{C}$, sectioned, and sections washed 3 times for $10 \mathrm{~min}$ in Tris buffered saline (TBS), then for $1 \mathrm{~h}$ at $4^{\circ} \mathrm{C}$ in TBS/3\% normal donkey serum $/ 0.1 \%$ Triton, incubated overnight at $4^{\circ} \mathrm{C}$ with rat antibodies recognizing dopamine transporter N-terminal sequences (MAB369, Millipore) diluted 1:1,000 in TBS/1\% normal donkey serum $/ 0.1 \%$ Triton, washed three times for $10 \mathrm{~min}$ in TBS, incubated for $2 \mathrm{~h}$ at $22^{\circ} \mathrm{C}$ with Alexa Fluora 488-conjugated donkey anti-rat IgG (A-21208, Life Technologies) diluted 1:2,400 in TBS/1\% normal donkey serum/0.1\% Triton, washed 3 times for $10 \mathrm{~min}$ in TBS and mounted onto slides using Prolong Gold (P-36931, Life Technologies). Tiled confocal immunofluorescence projection images from 11-level z stack images ( $1 \mu \mathrm{m}$ total) were produced from each section using a Zeiss 710 microscope (40× objective, excitation 488 and emission $519 \mathrm{~nm}$ ). The number of pixels that were members of groups of at least 100 contiguous pixels in which dopamine transporter (DAT) immunofluorescence was greater than two standard deviations above the mean value for each section was quantitated using ImageJ (version 1.48, NIH) by an observer unaware of genotype.

Mouse mRNAs were prepared and quantitated as noted for the human specimens from regional brain samples rapidly dissected from mouse brains. Expression of CDH13 mRNA in conditional knockouts was examined one week after CPP testing using oligonucleotides flCDH13f - flCDH13r anchored in the exon framed by the loxP sequences (exon 3) and CDH13af - CDH13ar, anchored in exons 7 and 8 (sequences available on request). Levels of mRNA corresponding to most mouse genes were compared using Affymetrix mouse gene 2.0 ST arrays, reagents recommended by the manufacturer (http:/ / www.affymetrix. com/estore/catalog/131477/AFFY/ Mouse+Genome+430+2.0+ Array\#1_3), 
a $30007 \mathrm{G}$ scanner and software AGCC v3.0, expression console v1.3 and transcriptome analysis v2.0.

Statistical analyses from mouse behavioral data used analyses of covariance (ANCOVA) with "PASW" statistics 18 (SPSS). We use age as a cofactor and genotype, dose and gender as between subjects factors and report influences of age and gender as well as interactions, below which they reach significance. Secondary analyses used Scheffe's post hoc and Student $t$ tests (Excel). Saccharin preferences were analyzed using factorial ANOVA with between-subjects factors of genotype, sex and cocaine dose. Neurochemical data were expressed as pg/mg wet weight, including data for monoamines, metabolites and metabolite-to-parent ratios: [DOPC+HVA]/dopamine and 5-HIAA/5-HT. Data were analyzed using two-way ANOVA (genotype and sex). Averaged densities of dopamine transporter immunoreactive elements in projected images of six sections from five mice of each genotype were compared using $t$ tests. Mouse expression array data was analyzed for triplicate arrays for each sample, three samples per genotype, using Gene Chip Operating Software v1.1.1 and a threshold of $>$ two-fold difference in expression. Correlations between SNP genotypes and levels of expression in human postmortem samples used the association analysis toolset PLINK (26). Bonferroni corrections for multiple testing with observed, average 0.4 correlations $(r)$ between CDH13 SNPs tested were performed using software available at (http:/ /www.quantitative skills.com/sisa/calculations /bonfer.php).

All supplementary materials are available online at www.molmed.org.

\section{RESULTS}

We report results from mice with constitutively altered and with adult (via conditional knockout) reductions in levels of CDH13 mRNA. We test these mice using CPP, a model of rewarding influences from drugs that has been highly validated in pharmacological studies (18) and supported by results of several molecular genetic studies (see below). We identify evidence for shifted dose response relationships for cocaine reward in constitutive $\mathrm{CDH} 13$ knockouts and in mice with reduced adult expression. These data fit with human observations that support influences of CDH13 variation on addiction-related phenotypes, and with the idea that drugs that modify activities of $C D H 13$, might be useful when given to adults.

We contrast these data with results of evaluations of other phenotypes that are of interest for several reasons. These include tests of motor abilities (reductions might confound CPP results), learning/ memory (reductions might confound CPP results), aversive features caused by cocaine (which might provide an alternative explanation for CPP results to "altered reward"), impulsivity (which might fit with ADHD influences but confound CPP results) and anxiety (which might confound CPP results). Results of these tests identify no obvious alternative or confounding behavioral explanation for these CPP differences. We report more subtle influences of $C D H 13$ knockout on ADHD-related tests of locomotion in male mice and on performance during early acquisition trials for complex tasks.

We test levels of monoamines in brains of the knockout mice as one possible contributor to the observed behavioral differences, and also test mRNA expression differences. In CDH13 cerebral cortices, we find selectively altered dopamine/metabolite ratios, differences in dopaminergic fiber densities and changes in an activity-dependent transcription factor mRNA. Each of these observations fits with CDH13 expression by dopaminergic neurons and subsets of cerebral cortical neurons and with contributions of changed dopaminergic innervation to the observed behavioral data.

\section{Human CDH13 Variation Versus Expression Levels}

CDH13 mRNA was detected in RNA extracted from cerebral cortical specimens from each of 184 individuals. There was good $(r>0.85)$ agreement between results obtained with amplimers targeting different parts of this mRNA. Levels of $\mathrm{CDH} 13$ expression were significantly associated with allele frequencies for SNPs in CDH13 intron 2 (Table S4). Nearby intron 2 SNPs, rs8059696, rs4783277, rs12596958 and rs2199430, displayed minor allele frequencies $\mathrm{ca}$ 0.35 , span about $8 \mathrm{~kb}$ and displayed nominally significant, $0.045>p>0.001$ association with levels of CDH13 expression. Brains from individuals with rs2199430 = GG displayed $181 \%$ of the mean levels of CDH13 mRNA found in AA homozygotes (Supplementary Figure S1; $p=0.0007$ ANOVA; Bonferroni-corrected threshold $p=0.003$ ).

\section{Mouse CDH13 Expression}

Heterozygote constitutive knockouts expressed approximately $50 \%$ of the wildtype levels of $C D H 13$ mRNA. Homozygote knockouts did not express detectable levels of this RNA (Supplement, Supplementary Figure S2A). Individual conditional knockouts sacrificed following CPP testing revealed varying levels of cerebral cortical expression of CDH13 mRNA (Supplementary Figure S2B; see below).

\section{Cocaine CPP in Constitutive CDH13 Knockout Mice}

Knockouts displayed altered dose/ response relationships for cocaine CPP (Figure 1A, $p=0.002$ for genotype*dose interaction, ANCOVA with age as covariate). In wild-type mice, 5, 10 and $20 \mathrm{mg} / \mathrm{kg}$ doses of cocaine induced significant place preferences (Scheffe's $p=0.035,<0.001$ and $<0.001$, respectively). In wild-type animals, significantly more robust preference was produced by 10 than by $5 \mathrm{mg} / \mathrm{kg}$ doses $(p=0.006)$. By contrast, homozygous $C D H 13$ knockout mice displayed significant preferences only for places paired with $5 \mathrm{mg} / \mathrm{kg} \mathrm{co}-$ caine $(p=0.002)$. Preferences induced by pairing with 10 or $20 \mathrm{mg} / \mathrm{kg}$ doses did not differ significantly from values in control, saline-injected animals ( $p=0.38$ and 0.10$)$. Heterozygous mice 


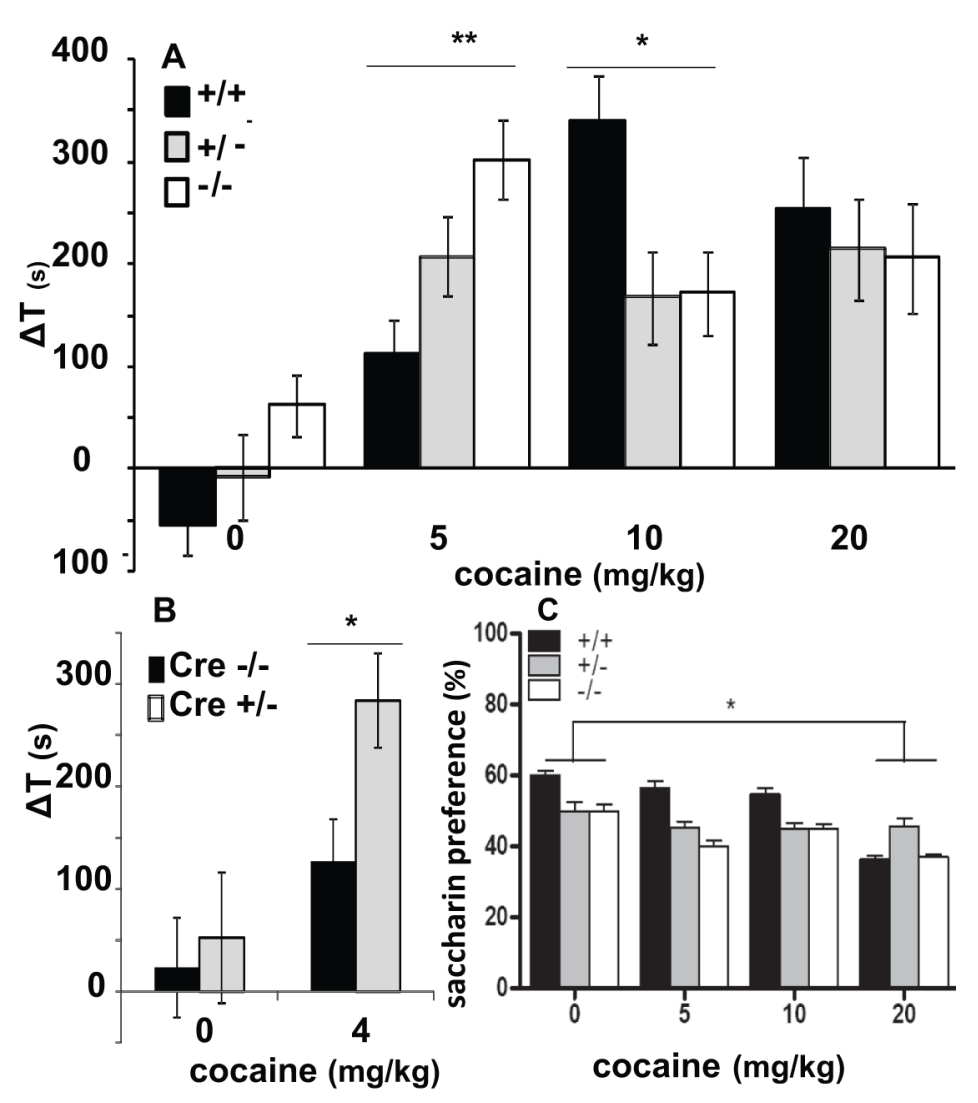

Figure 1. Constitutive (A, C) and adult (B) $\mathrm{CDH} 13$ knockout alters conditioned place preference $(A, B)$ but not conditioned taste aversion $(C)$ provided by modest to moderate cocaine doses. (A, B) Mean difference \pm SEM in time spent on the cocaine-paired side before and after conditioning ( $n=1-12$ /group). There was no significant difference between genotypes in time spent on the drug-paired side during the pre-tests for constitutive (ANOVA, $p=0.740$ ) or conditional ( $p=0.542$ ) knockouts. (C) Preference for cocaine-paired saccharin solution in two bottle testing after taste aversion conditioning. ${ }^{*} p<0.05,{ }^{* *} p<0.01$.

provided intermediate preferences for the places paired with 5,10 and $20 \mathrm{mg} / \mathrm{kg}$ cocaine doses that achieved statistical significance (Scheffe's $p=0.006,0.029$ and 0.007). Mice with reduced $\mathrm{CDH} 13$ expression thus display reduced reward from a normally highly rewarding $10 \mathrm{mg} / \mathrm{kg}$ cocaine dose (ANOVA $p=0.012$ ). They also display even more significant increases in preference for places paired with the $5 \mathrm{mg} / \mathrm{kg}$ dose (ANOVA $p=0.003$ ). These data suggest a leftward shift of the initial limb of the inverted U doseresponse relationship typically noted for cocaine reward and reinforcement. Dose-response relationships were similar in male and female mice (ANCOVA sex ${ }^{*}$ enotype*dose, $p=0.633$ ).

\section{Cocaine CPP in Conditional CDH13 Knockout Mice}

Mice in which expression was reduced in adulthood also increased preference for places paired with modest doses of cocaine (Figure 1B, ANCOVA genotype ${ }^{*}$ dose, $p=0.017 ; \mathrm{n}=11-12$ /group). Cre expressing mice showed significantly stronger preferences for places paired with $4 \mathrm{mg} / \mathrm{kg}$ cocaine than the control $\mathrm{Cre}_{-/ \mathrm{-}}$ mice $(p=0.018)$. There was a $r=-0.42$; one-tailed $p=0.035$ correlation between preference for the place paired with this modest cocaine dose and levels of
CDH13 mRNAs assessed in cortices of mice sacrificed following the conditioned place preference testing (Supplementary Figure S2B).

\section{Cocaine-Conditioned Taste Aversion}

There were no significant influences of genotype on acquisition of the taste aversion. In two-bottle tests (Figure 1C), there were significant effects of genotype and dose ( $p=0.029$ and 0.009 , respectively) but no significant genotype ${ }^{*}$ dose interaction $(\mathrm{p}=0.30)$. Conditioning with $20 \mathrm{mg} / \mathrm{kg}$ of cocaine significantly reduced saccharin preference in mice of all genotypes $(p=0.006)$.

\section{Locomotion}

During the first exposure to the $49 \times 49 \mathrm{~cm}$ arena, ANCOVA identified a $p=0.023$ effect of genotype on locomotion. Genotype effects were significant when data from males were analyzed separately (Supplementary Figure S3A, $p=0.034$ ) but not when data from females was analyzed separately (Supplementary Figure S3B, $p=0.265$ ).

During the first preconditioning session in the CPP apparatus (Supplementary Figure S4A), there was a significant genotype*age interaction (ANCOVA, $p=0.045)$. Constitutive $C D H 13$ knockout mice older than $90 \mathrm{~d}$ displayed less locomotion than their wild type siblings (ANOVA, $p=0.018$ ). In mice $<90 \mathrm{~d}$ old, genotype effects on locomotion did not reach significance (ANOVA, $p=0.095$ ).

There was no significant effect of genotype on locomotion monitored when mice were confined to halves of the conditioned place preference apparatus after treatments with saline, 5,10 or $20 \mathrm{mg} / \mathrm{kg}$ cocaine doses (Supplementary Figure S4B; ANCOVA, $p=0.15,0.13$ and 0.09, respectively; ANCOVA overall effect of genotype, $p=0.31$, genotype ${ }^{*}$ dose interaction, $p=0.88$ ).

\section{Rotarod Testing}

Knockout mice displayed a significant overall influence of genotype on the arc of learning this task (repeated measures ANCOVA day of test*genotype interaction, $p=0.040$ ) (Figure 2A). 

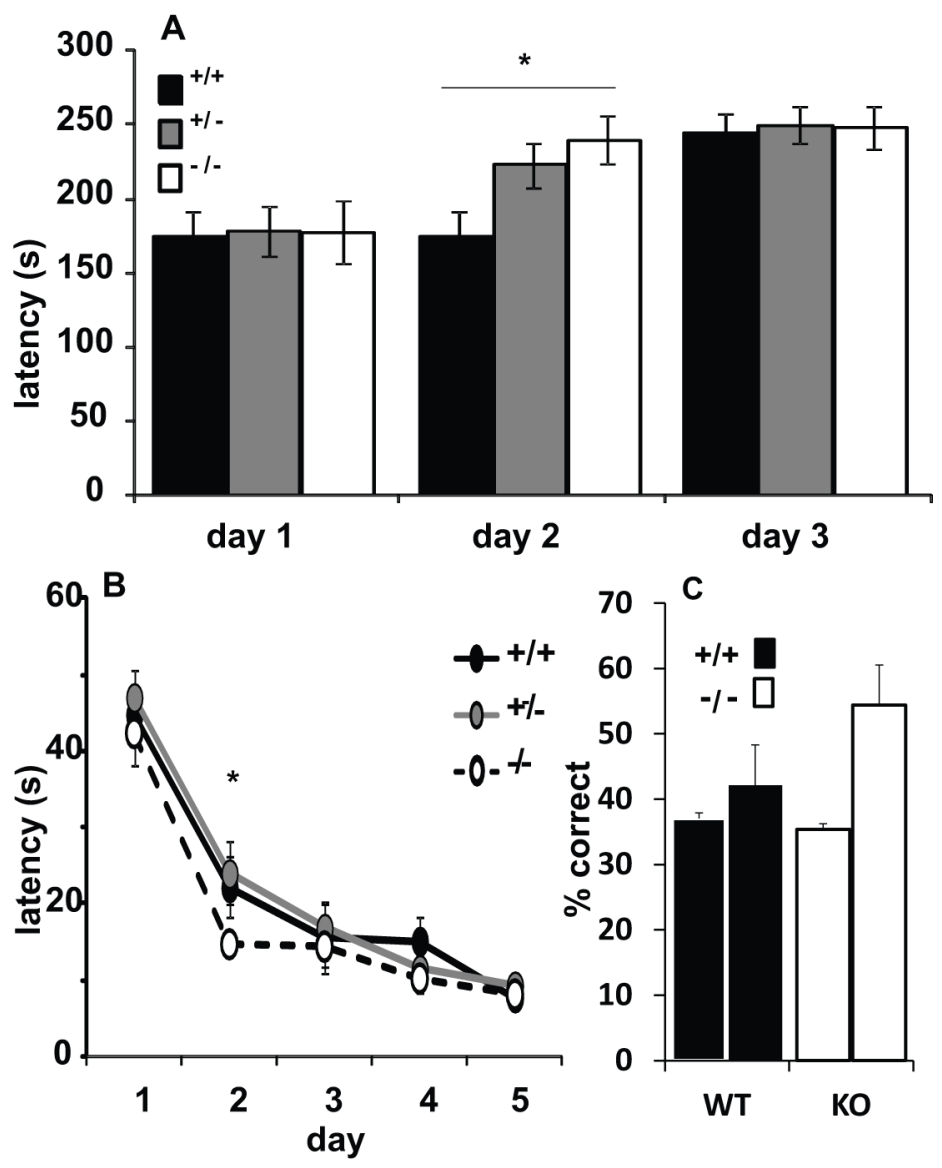

Figure 2. Acquisition and performance of the rotorod (A) Morris water maze (B) and 5-choice serial reaction time $(C)$ tasks in constitutive $C D H 13$ knockouts and wild-type littermates. $(A)$ Mean \pm SEM of the latencies to fall from the accelerating rotorod in wild-type, heterozygous and homozygous constitutive $\mathrm{CDH} 13$ knockouts. Knockouts showed faster acquisition of the task (repeated measures ANCOVA, $p=0.040$ ). $N=24 /$ genotype. ${ }^{*} p<0.05$. (B) Mean \pm SEM of the latencies to reach Morris water maze platform for wild-type, heterozygous and homozygous $C D H 13$ knockouts. Knockouts displayed no significant deficit in task acquisition (days $1-5$, repeated measures ANOVA genotype*day effect, $p=0.96$ ), and displayed better performance on d 2 of testing when compared with mice of both other genotypes * $p<0.05$.

(B) Mean \pm SEM of the probe trial results. (C) Mean \pm SEM of \% correct responses in 5-choice serial reaction time testing in the first 2 trials of this task in male mice. Knockouts showed a trend toward faster acquisition of the task that did not achieve significance $(p=0.1)$. Females failed to display any trends (data not shown).

This influence was greatest during the second test day (ANVOVA, effect of genotype, $p=0.017$ ), when knockouts performed better than wild-type littermates.

\section{Morris Water Maze}

Knockout mice displayed no significant deficit in Morris water maze tests of acquisition (Figure 2B; repeated measures ANCOVA genotype*day, $p=0.96$ ), reversal learning (repeated measures ANCOVA genotype*day, $p=0.81$ ), the first probe trial (Supplementary Figure S5B; including distance from the former platform location ANOVA, $p=0.13$; time spent searching in the target quadrant $p=0.12$; time spent searching in the opposite quadrant, $p=0.32$; and swimming speed, $p=0.45$ ) or the second probe trial after the platform had been moved to a new location. On the contrary, there was modest support for more rapid acquisition of the task in the knockouts, when compared to mice of both other genotypes (Figure 2B, $p=0.039$; $t$ test).

Five choice serial reaction time: Knockout mice displayed no significant deficit in 5-choice serial reaction time testing (Figure 2C, Supplementary Figure S6). During the first and second days of sessions in this apparatus, knockout male mice displayed 35 and $54 \%$ correct responses, while their wild-type littermates displayed 37 and $42 \%$ correct (Figure $2 \mathrm{C}$ ), though these differences failed to achieve statistical significance $(p=0.1)$. Knockout mice made no more premature responses or omission errors than wild-type littermates (Supplementary Figure S6).

\section{Anxiety}

CDH13KO mice did not differ from their wild-type siblings in latencies to emerge from a dark compartment or time spent in the light (Supplementary Figure S7A; ANCOVA effect of genotype, $p=0.2)$. There were no significant differences in the amount of time that they spent in the center of an open field (Supplementary Figure S7B; ANCOVA effect of genotype, $p=0.75$ ).

\section{Mouse Brain Neurochemistry}

In cortex, levels of dopamine were significantly reduced in constitutive $\mathrm{CDH} 13$ knockout mice (Figure 3A; ANOVA, $p=$ 0.006). Ratios between dopamine and its metabolites also differed significantly (Figure 3D; ANOVA, $p=0.007$ ). However, there were no significant differences in levels of dopamine, HVA or DOPAC in striatum, ventral midbrain or hippocampus (Figures 3B, C). There were no significant genotype-related differences in levels of norepinephrine, serotonin or its metabolite 5HIAA in cortex, striatum, hippocampus or ventral midbrain.

\section{Cortical Dopamine Transporter (DAT) Immunohistochemistry}

Infralimbic and frontal cortical regions displayed densities of DAT-immunoreactive elements in fiber/varicosity patterns 

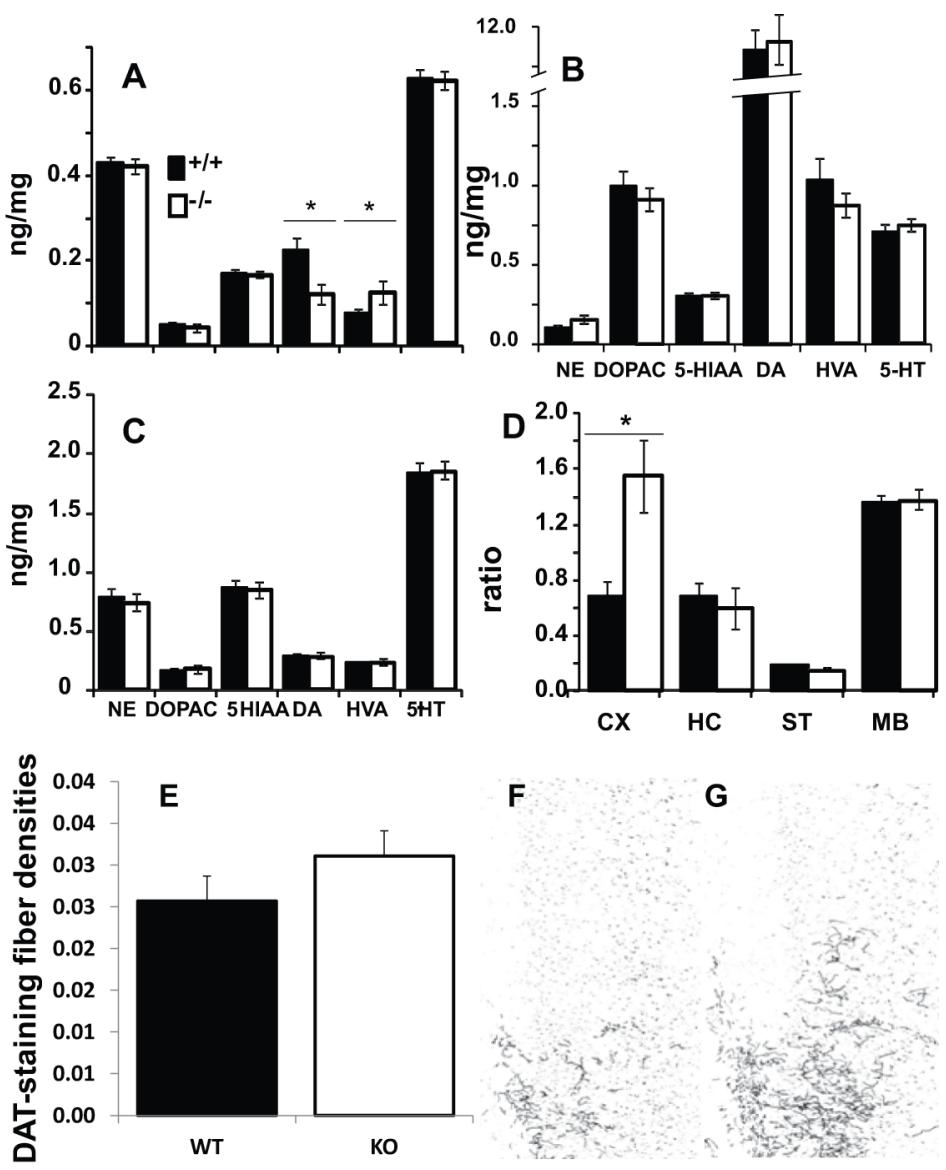

Figure 3. Altered dopamine levels $(A-D)$ and dopamine fiber densities $(E-G)$ in cortices of constitutive CDH13 knockout mice. Regional concentrations of monoamines and metabolites in dopamine-associated brain regions. ( $A-C$ ) Mean $\pm S E M$ of the concentrations of monoamines and metabolites in cerebral cortex (A), striatum (B) and ventral midbrain (C) samples dissected from brains of wild-type (black bars) and $C D H 13$ knockout (open bars) mice. (D) Ratios between dopamine metabolites and dopamine in: CX, cerebral cortex; HC, hippocampus; ST, striatum; MB, ventral midbrain. $N=10$ / genotype. ${ }^{*} p<0.05$. (E) Densities of dopamine transporter immumoreactive dopamine fibers in $50 \mu \mathrm{m}$ sections through prefrontal cortex from constitutive CDH13 knockouts and wild-type littermates. ${ }^{*} p<0.05$. (F-G) Images of dopamine transporter immunoreactive elements $>2$ SD above mean pixel density for each section from infralimbic/prefrontal cortical sections from wild-type (F) and CDH13 knockout $(G)$ mice. Midline is at the left in both images.

that were indistinguishable from those reported using several anti-DAT sera and material from several species $(27,28)$. Knockout mice displayed $1.3 \times$ the density of dopamine transporter immunoreactivity displayed by wild-type littermates ( $p=0.005, t$ test; Figures $3 \mathrm{E}, \mathrm{F})$.

\section{DISCUSSION}

CDH13 is a cell adhesion molecule expressed by neurons in brain circuits that include ventral midbrain substantia nigra and ventral tegmental area neurons, likely dopaminergic, whose activities are modulated by virtually every abused substance and by most ADHD pharmacotherapeutics (29-31). Homotypic interactions between CDH13 molecules on adjacent surfaces of expressing neurons are likely to alter the ways in which dopaminergic and other brain circuits that are implicated in addiction and motor modulation develop and are modified by pharmacological and physiological challenges (1,32-34).

The evidence for cis-regulation of CDH13 expression in humans identified in the current work adds to the posterior probabilities that $\mathrm{CDH} 13$ variation does alter addiction and ADHD-related phenotypes. The robust, $\mathrm{ca} .80 \%$ differences in cerebral cortical expression of CDH13 mRNA that we identify between individuals with common 5' CDH13 genotypes provides a link between data from the knockouts and common human allelic functional variation at this gene locus. SNPs in this $8 \mathrm{~kb} C D H 13$ intron 2 genomic region display nominally significant associations with addiction-related phenotypes; 9 of 17 and 8 of 20 tested SNPs display $0.05>p>2 \times 10^{-6}$ $\mathrm{p}_{\text {min }}$ associations with abilities to quit smoking or dependence on an addictive substance, repsectively (36). The intron 2 CDH13 SNP that displays the strongest association with level of CDH13 mRNA expression lies within $2 \mathrm{~kb}$ of a cluster of CDH13 SNPs that display the strongest associations with human temporal lobe volume (35). Mutually supporting positive and negative human and mouse data thus suggest that common $\mathrm{CDH} 13$ alleles that alter levels of expression in ways that provide links between individual differences identified in humans and differences that we report here in mouse models.

Characterization of mice with lifelong alterations in CDH13 expression supports a pattern of specific behavioral changes. Constitutive CDH13 knockout mice display alterations in their preference for the places paired with several different doses of cocaine. There is no corresponding influence of reduced CDH13 expression on the aversive features provided by these same cocaine doses, as assessed by conditioned taste aversion (36). There is no evidence for differences in anxiety phenotypes, which might otherwise interact with CPP testing (37). Constitutive knockout mice fail to display sizable alterations in locomotion or strength likely to confound CPP testing $(38,39)$. 
Effects on preference for places paired with a modest cocaine dose were also observed in conditional knockouts in which expression of CDH13 was changed only in adulthood. Developmental alterations in circuitry thus do not appear to be required to provide these most significant effects of altered CDH13 expression. We focused on influences of $4 \mathrm{mg} / \mathrm{kg}$ doses since these provided the largest differences in initial studies of these conditional knockouts. The significant correlations between mouse-to-mouse differences in levels of cortical CDH13 mRNA and preferences for places paired with $4 \mathrm{mg} / \mathrm{kg}$ doses do provide evidence for a graded relationship between levels of adult CDH13 expression and preference for this modest cocaine dose. It is conceivable that effects on reward from larger (e.g., $10 \mathrm{mg} / \mathrm{kg}$ ) cocaine doses might require lifelong reductions in CDH13 expression levels, however.

Subtle knockout influences on locomotion and cognitive performances reach significance in several interesting ways. Knockouts overall, and males analyzed separately, display significant differences in locomotion in a larger dark chamber. Knockouts of both genders display better performance during their second exposures to rotarod and Morris water maze testing. Each of these observations fit with reported modest human $\mathrm{CDH} 13$ associations with ADHD, which is more frequently diagnosed in males (40). However, the sizes of these effects are small, as are the modest effects noted by others in some mnemonic parameters of the conditional knockout mice (20). There are also no effects of constitutive changes in $\mathrm{CDH} 13$ on trained performance on the 5-choice serial reaction time task; the same result came from conditional CDH13 knockouts (20). The impulsivity phenotype assessed by this test (41) is thus not changed with $\mathrm{CDH} 13$ deletion.

Molecular, neurochemical and anatomic findings from $\mathrm{CDH} 13$ knockout mice provide evidence for specificity that complements behavioral results. Initial neurochemical studies highlighted specific changes in dopamine and its metabolites in cerebral cortex. These findings contrasted with lack of significant knockout versus wild-type differences in levels of other monoamines in any brain region sampled, or of dopamine in ventral midbrain or striatum. Neurochemical findings in cortex were then supported by changes in densities in dopamine-transporter immunoreactive fibers using a pixel based method; we obtained similar observations in initial studies using counts of labeled fibers by paired independent observers who were unaware of genotype. The greater fiber densities noted in knockouts and the elevated dopamine turnover (42) suggested by the altered ratios between dopamine and its major metabolites are each consistent with in vitro observations that CDH13-CDH13 interactions can inhibit outgrowth of $\mathrm{CDH} 13$-expressing neuronal processes (34). Microarray results that point to substantial changes in cortical expression of only the activity-

dependent transcription factor Npas4 (43) comport with both subtly altered cortical circuitry in the CDH13 knockouts and with the substantial specificity of the changes that $\mathrm{CDH} 13$ deletion induces.

Evidence available prior to and following our CPP results also supports differences in dose response relationships for addictive substances in individuals with differences in $\mathrm{CDH} 13$ genotypes (44). Prior to these mouse results, there were reports of associations between CDH13 markers and smoking quantity/ frequency (7) and dose-response relationships for symptoms of intoxication during individuals' first five exposures to alcohol (45). After we had obtained the initial CPP data from constitutive knockouts, Hart and colleagues reported associations between $\mathrm{CDH} 13$ variants and human individual differences in the dose-response relationships for amphetamine effects (16). There was a robust CDH13 association with individual differences in subjective positive responses to oral administration of $10 \mathrm{mg}$, but not $20 \mathrm{mg}$ amphetamine doses.
Evidence from mouse studies that CDH13 variation is associated with both cognitive and motor differences that could alter vulnerability to ADHD fits with human ADHD associations that were largely available as we performed the mouse experiments. The differences in locomotion that we identify here display interactions with gender that are reminiscent of the influences of gender on ADHD diagnoses and/or symptomatology. CDH13 associations with cognitive function in children with ADHD or autism fit nicely with the influences of CDH13 variation on task acquisition that we note here (46-48). We failed to identify any significant influences of $\mathrm{CDH} 13$ variation on the sorts of impulsivity measured by 5 -choice serial reaction time testing (49), although this "impulsivity" indicator may not assess all forms of impulsivity present in human disorders. Nevertheless, CDH13 variation does appear to provide subtle influences on both cognitive and motor systems in ways that could (subtly) alter likelihood of obtaining an ADHD diagnosis in several ways.

Our observations of altered cerebral cortical dopamine/metabolite levels and ratios in the $C D H 13$ knockouts provide a plausible neurochemical correlate for at least some of the specific influences of the knockout on drug reward. Homomeric recognition of $\mathrm{CDH} 13$ expressed by ventral tegmental area mesocortically projecting dopamine neurons and by many of the frontal cerebral cortical neurons that they target is likely to alter connections between these two important nodes of addiction-related circuitry.

Results from mice with altered $\mathrm{CDH} 13$ expression are reinforced by data from human associations that were available both prior to and following availability of the initial mouse CPP results. Prior probabilities for influences of $\mathrm{CDH} 13$ variation on addiction and ADHD phenotypes in mice were enhanced by findings, in several addiction and some ADHD association datasets, that clusters of nearby CDH13 SNPs display nominally significant $10^{-2}>p>10^{-8}$ 
case versus control allele frequency differences $(5,7,9,10,14,16,45,50-58)$. Cautions in interpreting this human data, taken alone, included the lack of large effects of any single CDH13 variant. Nevertheless, prior datasets, subsequent addiction-related studies and a meta-analysis of ADHD data (59) that do identify CDH13 supported elevated prior probabilities that $\mathrm{CDH} 13$ associations would be identified in the current work. In light of these prior probabilities, the robust influences of CDH13 knockout on stimulant-conditioned place preference and modest influences on motor/learning phenotypes that are consistent with ADHD associations elevate posterior probabilities that $\mathrm{CDH} 13$ variation does provide bona fide influences on addictionand ADHD-related phenotypes.

CDH13 variation, implicated in addiction, in rewarding and/or aversive responses to stimulants and alcohol and in ADHD by human molecular genetic studies, provides a pattern of altered behaviors in a mouse model that accords remarkably with human data for these phenotypes. In future work, it will be interesting to see if mice with reduced CDH13 expression display differences in responses to natural rewards and/or reward from other substances of abuse. Nevertheless, the present results support the important roles that detailed patterns of neuronal connections (6), including those modulated by $C D H 13$, are likely to play in addiction and other complex brain phenotypes. These data add to support for $\mathrm{CDH13}$ as a candidate druggable target for therapies for addictions and/or ADHD (60).

\section{CONCLUSION}

Few of the likely polygenic influences on human vulnerabilities to addiction or attention deficit hyperactivity disorder (ADHD) have been elucidated in detail or in animal models. Mice with lifelong reductions in expression of cadherin 13 (CDH13) display sizable differences in tests of stimulant reward, modest/ gender-specific influences on locomotor behaviors (though not 5-choice testing) of possible relevance to ADHD and altered cortical dopamine systems that could contribute to these behavioral observations. Mouse and human data are linked by differences in $\mathrm{CDH} 13$ mRNA expression in brains of humans with different $\mathrm{CDH} 13$ genotypes and by human associations of CDH13 variants with addiction, stimulant reward and ADHD phenotypes. These data and altered cocaine reward in mice with adult reductions in $\mathrm{CDH} 13$ expression support $\mathrm{CDH13}$ as a novel therapeutic target.

\section{ACKNOWLEDGMENTS}

We are grateful for help from C Johnson, D Arking, D Naimen, J Bader, M Morales and J Schroder, for access to CDH13 association data from A Hart, A Palmer, O Kohannim and $\mathrm{P}$ Thompson and for access to brain samples from the University of Maryland Brain Tissue Bank. Correspondence concerning CDH13 knockout mice to BR, at $10901 \mathrm{~N}$. Torrey Pines Road, La Jolla, CA 92037.

\section{DISCLOSURE}

This work was supported by the National Institutes of Health (NIH)Intramural Research Program, NIDA, DHHS and the Biomedical Research Institute of New Mexico (G Uhl), NIH grant HL102680 (B Ranscht), the European Community (EC: AGGRESSOTYPE FP7/No. 602805 (OR and KPL), the DFG (SFB TRR 58/A5 OR and KPL) and the Fritz Thyssen Foundation (Az.10.13.1185 OR and KPL).

\section{REFERENCES}

1. Ranscht B, Dours-Zimmermann MT (1991): T-cadherin, a novel cadherin cell adhesion molecule in the nervous system lacks the conserved cytoplasmic region. Neuron. 7:391-402.

2. Vestal DJ, Ranscht B (1992): Glycosyl phosphatidylinositol-anchored T-cadherin mediates calcium-dependent, homophilic cell adhesion. J. Cell Biol. 119:451-61.

3. Saddoris MP, Sugam JA, Cacciapaglia F, Carelli RM (2013): Rapid dopamine dynamics in the accumbens core and shell: learning and action. Front Biosci (Elite Ed). 5:273-88.

4. Lein ES, Hawrylycz MJ, Ao N, Ayres M, Bensinger A, Bernard A, et al. (2007): Genome-wide atlas of gene expression in the adult mouse brain. Nature. 445:168-76.
5. Treutlein J, Cichon S, Ridinger M, Wodarz N, Soyka M, Zill P, et al. (2009): Genomewide association study of alcohol dependence. Arch. Gen. Psychiatry. 66:773-84.

6. Uhl GR, Drgon T, Johnson C, Li CY, Contoreggi C, Hess J, et al. (2008): Molecular genetics of addiction and related heritable phenotypes: genome-wide association approaches identify "connectivity constellation" and drug target genes with pleiotropic effects. Ann NY Acad Sci. 1141:318-81.

7. Thorgeirsson TE, Gudbjartsson DF, Surakka I, Vink JM, Amin N, Geller F, et al. (2010): Sequence variants at CHRNB3-CHRNA6 and CYP2A6 affect smoking behavior. Nat. Genet. 42:448-53.

8. Drgon T, Johnson CA, Nino M, Drgonova J, Walther DM, Uhl GR (2011): "Replicated" genome wide association for dependence on illegal substances: genomic regions identified by overlapping clusters of nominally positive SNPs. American Journal of Medical Genetics Part B Neuropsychiatric Genetics. 156:125-38.

9. Uhl GR, Drgon T, Liu QR, Johnson C, Walther D, Komiyama T, et al. (2008): Genomewide association for methamphetamine dependence: convergent results from 2 samples. Arch Gen Psychiatry. 65:345-55.

10. Johnson C, Drgon T, Walther D, Uhl GR (2011): Genomic regions identified by overlapping clusters of nominally-positive SNPs from genome-wide studies of alcohol and illegal substance dependence. PLoS One. 6:e19210.

11. Johnson C, Drgon T, Liu QR, Walther D, Edenberg H, Rice J, et al. (2006): Pooled association genome scanning for alcohol dependence using 104,268 SNPs: validation and use to identify alcoholism vulnerability loci in unrelated individuals from the collaborative study on the genetics of alcoholism. American Journal of Medical Genetics Part B Neuropsychiatric Genetics. 141B:844-53.

12. Liu QR, Drgon T, Johnson C, Walther D, Hess J, Uhl GR (2006): Addiction molecular genetics: 639,401 SNP whole genome association identifies many "cell adhesion" genes. American Journal of Medical Genetics Part B Neuropsychiatric Genetics 141B:918-25.

13. Johnson C, Drgon T, Liu QR, Zhang PW, Walther D, Li CY, et al. (2008): Genome wide association for substance dependence: convergent results from epidemiologic and research volunteer samples. BMC Med Genet. 9:113.

14. Drgon T, Montoya I, Johnson C, Liu QR, Walther D, Hamer D, et al. (2009): Genomewide association for nicotine dependence and smoking cessation success in NIH research volunteers. Mol. Med. 15:21-7.

15. Uhl GR, Walther D, Musci R, Fisher C, Anthony JC, Storr CL, et al. (2012): Smoking quit success genotype score predicts quit success and distinct patterns of developmental involvement with common addictive substances. Mol. Psychiatry.

16. Hart AB, Engelhardt BE, Wardle MC, Sokoloff G, Stephens M, de Wit H, et al. (2012): Genome-wide 
association study of $\mathrm{d}$-amphetamine response in healthy volunteers identifies putative associations, including cadherin 13 (CDH13). PLoS One. 7:e42646.

17. Joslyn G, Ravindranathan A, Brush G, Schuckit M, White RL (2010): Human variation in alcohol response is influenced by variation in neuronal signaling genes. Alcoholism Clinical and Experimental Research. 34:800-12.

18. Tzschentke TM (1998): Measuring reward with the conditioned place preference paradigm: a comprehensive review of drug effects, recent progress and new issues. Prog Neurobiol. 56:613-72.

19. Hebbard LW, Garlatti M, Young LJ, Cardiff RD, Oshima RG, Ranscht B (2008): Tcadherin supports angiogenesis and adiponectin association with the vasculature in a mouse mammary tumor model. Cancer Res. 68:1407-16.

20. Rivero O, Selten MM, Sich S, Popp S, Bacmeister L, Amendola E, Negwer M, Schubert D, Proft F, Kiser D, Schmitt AG, Gross C, Kolk SM, Strekalova T, van den Hove D, Resink TJ, Nadif Kasri N and Lesch KP (2015): Cadherin-13, a risk gene for ADHD and comorbid disorders, impacts GABAergic function in hippocampus and cognition Transl Psychiatry. 13;5:e655. doi: 10.1038/tp.2015.152.

21. Drgonova J, Zimonjic DB, Hall FS, Uhl GR (2010): Effect of KEPI (Ppp1r14c) deletion on morphine analgesia and tolerance in mice of different genetic backgrounds: when a knockout is near a relevant quantitative trait locus. Neuroscience. 165:882-95.

22. Hall FS, Centeno M, Perona MT, Adair J, Dobner PR, Uhl GR (2011): Effects of neurotensin gene knockout in mice on the behavioral effects of cocaine. Psychopharmacology (Berl).

23. Serafine KM, Riley AL (2009): Possible role of norepinephrine in cocaine-induced conditioned taste aversions. Pharmacol Biochem Behav. 92:111-16.

24. Morris R (1984): Developments of a water-maze procedure for studying spatial learning in the rat. J. Neurosci. Methods. 11:47-60.

25. Baumann MH, Ayestas MA, Jr., Partilla JS, Sink JR, Shulgin AT, Daley PF, et al. (2012): The designer methcathinone analogs, mephedrone and methylone, are substrates for monoamine transporters in brain tissue. Neuropsychopharmacology. 37:1192-203.

26. http://pngu.mgh.harvard.edu/ purcell/plink/

27. Freed C, Revay R, Vaughan RA, Kriek E, Grant S, Uhl GR, et al. (1995): Dopamine transporter immunoreactivity in rat brain. J Comp Neurol. 359:340-49.

28. Sesack SR, Hawrylak VA, Guido MA, Levey AI (1998): Cellular and subcellular localization of the dopamine transporter in rat cortex. Adv Pharmacol. 42:171-4.

29. Volkow ND, Wang GJ, Fowler JS, Tomasi D (2012): Addiction circuitry in the human brain. Annu Rev Pharmacol Toxicol. 52:321-36.

30. De Mei C, Ramos M, Iitaka C, Borrelli E (2009): Getting specialized: presynaptic and postsynap- tic dopamine D2 receptors. Curr Opin Pharmacol. 9:53-8.

31. Sinita E, Coghill D (2014): The use of stimulant medications for non-core aspects of ADHD and in other disorders. Neuropharmacology.

32. Ranscht B (2000): Cadherins: molecular codes for axon guidance and synapse formation. International Journal of Developmental Neuroscience. 18:643-51.

33. Philippova M, Joshi MB, Kyriakakis E, Pfaff D, Erne P, Resink TJ (2009): A guide and guard: the many faces of T-cadherin. Cell Signal. 21: 1035-1044.

34. Ciatto C, Bahna F, Zampieri N, VanSteenhouse HC, Katsamba PS, Ahlsen G, et al. (2010): T-cadherin structures reveal a novel adhesive binding mechanism. Nat Struct Mol Biol. 17:339-47.

35. Kohannim O, Hibar DP, Stein JL, Jahanshad N, Hua X, Rajagopalan P, et al. (2012): Discovery and Replication of Gene Influences on Brain Structure Using LASSO Regression. Front Neurosci. 6:115.

36. Verendeev A, Riley AL (2013): The role of the aversive effects of drugs in selfadministration: assessing the balance of reward and aversion in drug-taking behavior. Behav Pharmacol. 24:363-74.

37. Dennis TS, Beck KD, Bobzean SA, Dougall AL, Perrotti LI (2012): Assessing learned associations between conditioned cocaine reward and environmental stimuli in the Wistar Kyoto rat. Pharmacol Biochem Behav. 103:76-82.

38. Tyrberg B, Miles P, Azizian KT, Denzel MS, Nieves ML, Monosov EZ, et al. (2011): Tcadherin (Cdh13) in association with pancreatic beta-cell granules contributes to second phase insulin secretion. Islets. 3:327-37.

39. Williams AS, Kasahara DI, Verbout NG, Fedulov AV, Zhu M, Si H, et al. (2012): Role of the adiponectin binding protein, T-cadherin (Cdh13), in allergic airways responses in mice. PLoS One. 7:e41088.

40. Wittchen HU, Jacobi F, Rehm J, Gustavsson A, Svensson M, Jonsson B, et al. (2011): The size and burden of mental disorders and other disorders of the brain in Europe 2010. Eur Neuropsychopharmacol. 21:655-79.

41. Uhl G (2007): Premature poking: impulsivity, cocaine and dopamine. Nat. Med. 13:413-14

42. Murotani T, Ishizuka T, Hattori S, Hashimoto R, Matsuzaki S, Yamatodani A (2007): High dopamine turnover in the brains of Sandy mice. Neurosci. Lett. 421:47-51.

43. Benito E, Barco A (2014): The Neuronal Activity-Driven Transcriptome. Mol Neurobiol.

44. Uhl GR, Drgonova J, Hall FS (2014): Curious cases: Altered dose-response relationships in addiction genetics. Pharmacol. Ther. 141:335-46.

45. Joslyn G, Ravindranathan A, Brush G, Schuckit M, White RL (2010): Human variation in alcohol response is influenced by variation in neuronal signaling genes. Alcohol Clin Exp Res. 34:800-12.

46. Arias-Vasquez A, Altink ME, Rommelse NN, Slaats-Willemse DI, Buschgens CJ, Fliers EA, et al. (2011): CDH13 is associated with working memory performance in attention deficit/hyperactivity disorder. Genes Brain Behav. 10:844-51.

47. Chapman NH, Estes A, Munson J, Bernier R, Webb SJ, Rothstein JH, et al. (2011): Genome-scan for IQ discrepancy in autism: evidence for loci on chromosomes 10 and 16. Hum Genet. 129:59-70.

48. Wang K, Zhang H, Ma D, Bucan M, Glessner JT, Abrahams BS, et al. (2009): Common genetic variants on 5 p14.1 associate with autism spectrum disorders. Nature. 459:528-33.

49. Bari A, Robbins TW (2013): Inhibition and impulsivity: behavioral and neural basis of response control. Prog Neurobiol. 108:44-79.

50. Uhl GR, Liu QR, Drgon T, Johnson C, Walther D, Rose JE, et al. (2008): Molecular genetics of successful smoking cessation: convergent genome-wide association study results. Arch. Gen. Psychiatry. 65:683-93.

51. Uhl GR, Drgon T, Johnson C, Ramoni MF, Behm FM, Rose JE (2010): Genome-wide association for smoking cessation success in a trial of precessation nicotine replacement. Mol Med. 16:513-26.

52. Drgon T, Johnson C, Walther D, Albino AP, Rose JE, Uhl GR (2009): Genome-wide association for smoking cessation success: participants in a trial with adjunctive denicotinized cigarettes. Mol. Med. 15:268-74.

53. Uhl GR, Drgon T, Johnson C, Walther D, David SP, Aveyard P, et al. (2010): Genomewide association for smoking cessation success: participants in the Patch in Practice trial of nicotine replacement. Pharmacogenomics. 11:357-67.

54. Johnson C, Drgon T, Liu QR, Walther D, Edenberg H, Rice J, et al. (2006): Pooled association genome scanning for alcohol dependence using 104,268 SNPs: validation and use to identify alcoholism vulnerability loci in unrelated individuals from the collaborative study on the genetics of alcoholism. Am. J. Med. Genet B. Neuropsychiatr. Genet. 141B:844-53.

55. Drgon T, Johnson CA, Nino M, Drgonova J, Walther DM, Uhl GR (2011): "Replicated" genome wide association for dependence on illegal substances: genomic regions identified by overlapping clusters of nominally positive SNPs. Am. J. Med. Genet B. Neuropsychiatr. Genet. 156:125-38.

56. Liu QR, Drgon T, Johnson C, Walther D, Hess J, Uhl GR (2006): Addiction molecular genetics: 639,401 SNP whole genome association identifies many "cell adhesion" genes. Am J Med Genet B. Neuropsychiatr. Genet. 141B:918-25.

57. Lasky-Su J, Neale BM, Franke B, Anney RJ, Zhou K, Maller JB, et al. (2008): Genomewide association scan of quantitative traits for attention deficit hyperactivity disorder identifies novel associations and confirms candidate gene associations. Am. J. Med. Genet B Neuropsychiatr Genet. 147B:1345-54.

58. Lesch KP, Timmesfeld N, Renner TJ, Halperin R, Roser C, Nguyen TT, et al. (2008): Molecular 
genetics of adult ADHD: converging evidence from genome-wide association and extended pedigree linkage studies. J. Neural Transm. 115:1573-85.

59. Neale BM, Medland SE, Ripke S, Asherson P, Franke B, Lesch KP, et al. (2010): Metaanalysis of genome-wide association studies of attention-deficit/hyperactivity disorder. J Am Acad Child Adolesc Psychiatry. 49:884-97.

60. Uhl GR, Drgonova J (2014): Cell adhesion molecules: druggable targets for modulating the connectome and brain disorders? Neuropsychopharmacology. 39:235.

Cite this article as: Drgonova J, et al. (2016) Cadherin 13: Human cis-regulation and selectively altered addiction phenotypes and cerebral cortical dopamine in knockout mice. Mol. Med. 22:537-47. 